\title{
Halloysite reinforced epoxy composites with improved mechanical properties
}

\author{
Muhammad Jawwad Saif ${ }^{1 *}$, Muhammad Asif ${ }^{1}$, Muhammad Naveed ${ }^{1}$, Khalid Mahmood Zia ${ }^{1}$, \\ Waheed -uz- Zaman', Muhammad Kaleem Khosa ${ }^{3}$, Muhammad Asghar Jamal ${ }^{3}$ \\ ${ }^{1}$ Government College University Faisalabad, Department of Applied Chemistry, Pakistan \\ ${ }^{2}$ University of the Punjab, Institute of Chemistry, Lahore, Pakistan \\ ${ }^{3}$ Government College University Faisalabad, Department of Chemistry, Pakistan \\ "Corresponding author: e-mail: jawwadsaif@gcuf.edu.pk
}

\begin{abstract}
Halloysite nanotubes (HNTs) reinforced epoxy composites with improved mechanical properties were prepared. The prepared HNTs reinforced epoxy composites demonstrated improved mechanical properties especially the fracture toughness and flexural strength. The flexural modulus of nanocomposite with $6 \%$ mHNTs loading was $11.8 \%$ higher than that of neat epoxy resin. In addition, the nanocomposites showed improved dimensional stability. The prepared halloysite reinforced epoxy composites were characterized by thermal gravimetric analysis (TGA). The improved properties are attributed to the unique characteristics of HNTs, uniform dispersion of reinforcement and interfacial coupling.
\end{abstract}

Keywords: epoxy resin composites, halloysite nanotubes, polymers.

\section{INTRODUCTION}

Halloysite is a kind of aluminosilicate clay mineral with a tubular structure. The natural deposits of halloysite nanotubes are found in countries like China, New Zealand, America, Brazil, and France. HNTs are chemically related to kaolinite and are used in the fabrication of high quality ceramic white-wares. Researchers have done a lot of work on the characterization of the halloysite clay ${ }^{1}$. Lately it has been reported that HNTs have distinctive dimensional structure and these are formed by two building blocks (i) tetrahedral, in which sheets of corner are shared $\left[\mathrm{SiO}_{4}\right]$ and (ii) octahedral, in which sheets of edges are shared $\left[\mathrm{AlO}_{6}\right]^{2}$. Within the octahedral sheet, only two-third of the presented octahedral sites are filled with aluminium. The layers which make up the ultimate structure are poised by one of each of these sheets so that the crystal structure of halloysite is described as 1:1 dioctahedral layer silicate ${ }^{3}$. HNTs possess very advantageous characteristics such as nanoscale lumens, better length/diameter (L/D) ratio, less hydroxyl group density on surface. Researchers have been using these properties to formulate high quality ceramics ${ }^{4,5}$. There are increasing number of studies focusing on preparation of polymer/HNT nanocomposites and study of its properties. HNTs have been employed in fabrication of polyamide $6^{6,7}$, polypropylene ${ }^{8}$ and poly(butylene terephthalate) ${ }^{9}$ composites using a melt-blending technique. These thermoplastic composites showed superior mechanical properties as compared with neat polymers. Halloysite nanotubes are much cheaper as compared with carbon nanotubes. Therefore, we have chosen halloysite clay nanotubes as reinforcement in epoxy matrix.

\section{EXPERIMENTAL}

\section{Material}

The epoxy resin used was diglycidyl ether of bisphenol A, Araldite-F (Ciba, with epoxy content $5.25 \mathrm{Eq} / \mathrm{Kg}$ ). Bisphenol A dicyanate ester (Ciba, AroCy B-10) was used as hardener. HNTs were purchased from Natural Nano Inc. (USA). The Brunauer-Emmett-Teller (BET) surface area of HNTs was found to be $48.58 \mathrm{~m}^{2} / \mathrm{g}$. A coupling agent (Dow Corning Co., Ltd., $\gamma$-glycidoxypropyltrimet hoxysilane) was used in order to improve the interfacial interactions between the matrix and reinforcement.

\section{Purification and modification of HNTs}

A $10 \%(\mathrm{w} / \mathrm{w})$ water solution of halloysite nanotubes was prepared by introducing distilled water in dry halloysite (as received from supplier). Sodium hexametaphosphate $(0.03 \%)$ was added to aqueous halloysite solution. The solution was kept stirring for 30 minutes and left for standing for another 30 minutes. The impurities and some larger aggregates settled in the bottom. The upper layer was carefully separated. Halloysite nanotubes were recovered by centrifugation, dried at $75^{\circ} \mathrm{C}$ overnight.

Surface silylation was performed by reacting $\gamma$-glyc idoxypropyltrimethoxysilane with halloysite surface in acidic medium. Halloysite (10 g) was dispersed in water/ ethanol mixture (50:50). The $\mathrm{pH}$ value was adjusted to 5 by addition of acetic acid. Silane $(2 \mathrm{~g})$ was added to the halloysite dispersion and stirred for 24 hours. Modified halloysite were filtered, again dispersed in methanol, stirred for 1 hour and finally filtered and dried in air.

\section{Characterization of modified halloysite (m-HNTs)}

Modified halloysite was characterized by thermogravimetric analysis with SDT Q600 instrument at a heating rate of $10^{\circ} \mathrm{C} /$ minute from $50^{\circ} \mathrm{C}$ to $700^{\circ} \mathrm{C}$ in normal environment (air).

\section{Preparation of epoxy nanocomposite}

Modified halloysite (m-HNTs) were thoroughly dispersed in epoxy resin at $60^{\circ} \mathrm{C}$ using a stirrer. The mixture was degassed. Curing agent was added under stirring to mix it completely. The mixture was again degassed and poured into Teflon lined molds. The mixture was cured at $120^{\circ} \mathrm{C} / 3 \mathrm{~h}+180^{\circ} \mathrm{C} / 1 \mathrm{~h}$. Samples were prepared with varying content of modified halloysite in composite ranging from $3-9 \%$.

\section{Characterization of halloysite-epoxy composites}

An Instron 4465 Universal tester (ASTM D90) was used to determine the flexural properties. Tests were performed at ambient temperature by employing a cross-head speed of $2 \mathrm{~mm} /$ minute. Tests were performed in 
triplicate. A NETZSCH DIL 402 PC dilatometer was used to measure coefficient of thermal expansion (CTE) by employing a heating rate of $10^{\circ} \mathrm{C} /$ minute from room temperature to $180^{\circ} \mathrm{C}$.

\section{RESULTS AND DISCUSSION}

Halloysite nanotubes are found in agglomerated form owing to their hydrophilic nature. There might be some impurities (illite, quartz etc) present in commercial halloysite samples. It is necessary to purify the commercial HNTs of such impurities. After purification, HNTs were treated with $\gamma$-glycidoxypropyltrimethoxysilane in order to facilitate dispersion in matrix and to improve the matrix-reinforcement interfacial interaction. The silane was grafted on HNTs by condensation mechanism in acidic medium. $\gamma$-Glycidoxypropyltrimethoxysilane tends to hydrolyse in aqueous acidic solutions to produce silane molecules containing terminal hydroxyl groups, that is capable of condensing with $-\mathrm{OH}$ containing HNTs surface. Modified HNTs were characterized with TGA and the weight loss curves before and after modification confirmed successful grafting of silane (Fig. 1). The increased weight loss in modified HNTs can be attributed to decomposition of organic layer present on the surface of HNTs.

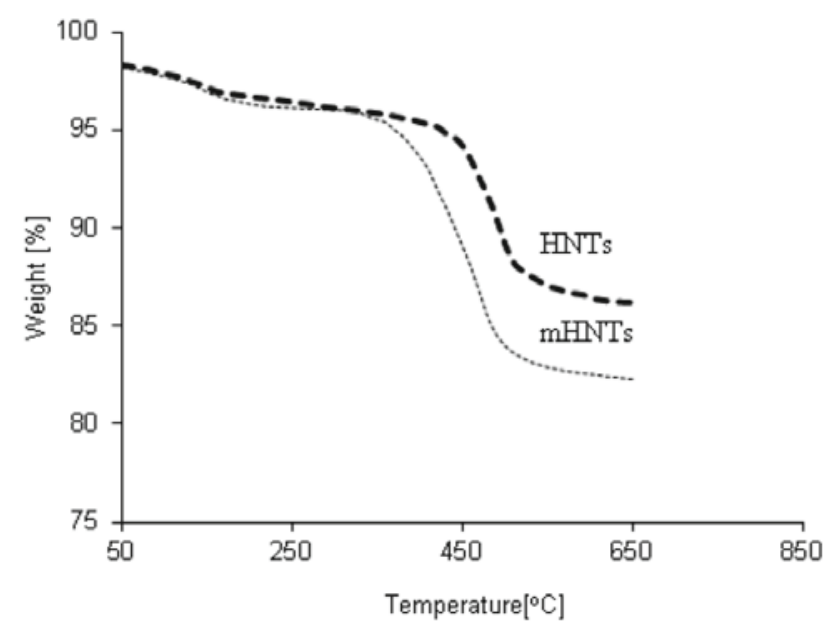

Figure 1. TGA of HNTs and modified HNTs

Mechanical properties of nanocomposites greatly depend on the dispersion state of filler. A better dispersion of filler results in improved mechanical properties whereas aggregated particles tend to adversely affect the mechanical properties. Modified HNTs have better compatibility with epoxy matrix and hence a more uniform dispersion was achieved.

Thermogravimetric analysis was performed to study thermal stability of halloysite reinforced epoxy composites (Fig. 2). It is obvious from TGA plots that thermal stability increases with an increase in HNTs content in epoxy. This effect is more pronounced at elevated temperatures. The degradation process involves dehydration followed by chain scission. This TGA analysis is in consistence with earlier reported effects of silicates as reinforcement. This data reveals that char yield increases with an increase in halloysite content in the composite and express flame retardant behavior of HNTs.

Coefficient of thermal expansion (CTE) is a crucial property of epoxies. Lower CTE presents high dimensional stability of polymers. Incorporation of inorganic fillers having very low CTE effectively lowers CTE of polymers. CTE values for mHNTs-epoxy composites are given in Table 1. The data reveals that CTE of mHNTsepoxy composites was decreased with addition of mHNTs. This could be explained in terms of better dispersion of mHNTs in polymer matrix, high dimensional stability of halloysite and improved interfacial interaction between mHNTs and epoxy matrix.

Figure 3 represents SEM micrograph of a cracked HNT-epoxy composite. The dispersion of HNTs in epoxy matrix is generally uniform with some exception of HNTs clusters. Finding of HNTs cluster is frequent in samples with high HNTs loading (10\% loading). Theoretically, high loading of HNTs can improve the mechanical properties in epoxy composites, however, large doses of HNTs result in particle clusters that could be detrimental to overall mechanical properties. Therefore, an optimal dose of HNTs produce the best results.

Flexural properties of HNTs-epoxy composites were evaluated to investigate the effect of HNTs on flexural behavior of epoxy resin. Data is presented in Table 2. It was observed that flexural strength and flexural modulus increased with an increase in HNTs quantity in composites. The flexural modulus of nanocomposite with $6 \%$ mHNTs loading is $11.8 \%$ higher than that of neat epoxy resin. There is little increase in flexural properties with further increase in halloysite content. Even further increase in HNT content can lead to particle agglomeration that adversely affects the flexural properties of composite material. Previous literature also suggests an

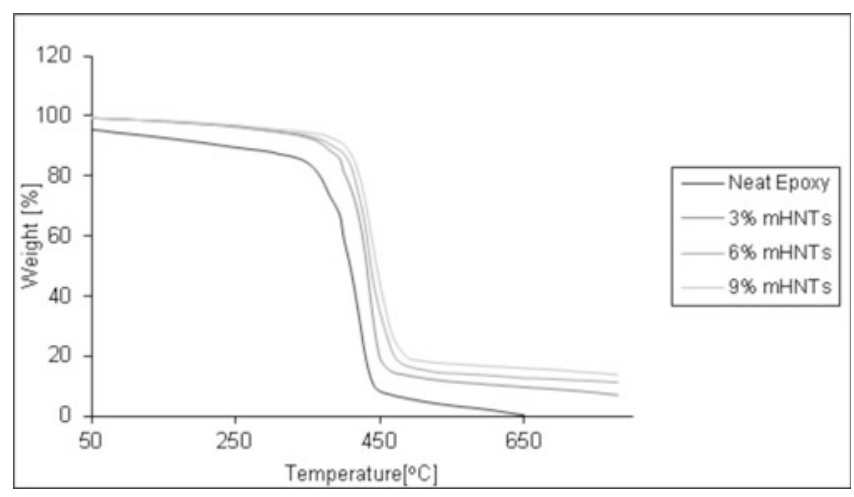

Figure 2. TGA of mHNTs - epoxy composites

Table 1. CTE of mHNTs-epoxy composites

\begin{tabular}{|l|c|c|c|}
\hline Sample & CTE $30-100^{\circ} \mathrm{C}\left[\mathrm{ppm} /{ }^{\circ} \mathrm{C}\right]$ & $\%$ Decrease & $\mathrm{CTE} 100-170^{\circ} \mathrm{C}\left[\mathrm{ppm} /{ }^{\circ} \mathrm{C}\right]$ \\
\hline Neat epoxy & 46.3 & - & 73.3 \\
\hline $1.0 \mathrm{wt} \% \mathrm{mHNTs}$ & 45.8 & 1.1 & 72.1 \\
\hline $2.0 \mathrm{wt} \% \mathrm{mHNTs}$ & 45.3 & 2.2 & 71.8 \\
\hline $3.0 \mathrm{wt} \% \mathrm{mHNTs}$ & 44.7 & 3.5 & 69.4 \\
\hline $6.0 \mathrm{wt} \% \mathrm{mHNTs}$ & 43.8 & 5.4 & 68.8 \\
\hline $8.0 \mathrm{wt} \% \mathrm{mHNTs}$ & 43.7 & 5.6 & 6.3 \\
\hline $10 \mathrm{wt} \% \mathrm{mHNTs}$ & 43.4 & 6.2 & 6.1 \\
\hline
\end{tabular}


Table 2. Flexural strength and flexural modulus of mHNTs-epoxy composites

\begin{tabular}{|l|c|c|}
\hline Samples & $\begin{array}{c}\text { Flexural strength } \\
{[\mathrm{MPa}]}\end{array}$ & $\begin{array}{c}\text { Flexural modulus } \\
{[\mathrm{GPa}]}\end{array}$ \\
\hline Neat epoxy & 38 & 2.88 \\
\hline $1.0 \mathrm{wt} \% \mathrm{mHNTs}$ & 53 & 3.04 \\
\hline $2.0 \mathrm{wt} \% \mathrm{mHNTs}$ & 69 & 3.07 \\
\hline $3.0 \mathrm{wt} \% \mathrm{mHNTs}$ & 76 & 3.14 \\
\hline $6.0 \mathrm{wt} \% \mathrm{mHNTs}$ & 89 & 3.22 \\
\hline $8.0 \mathrm{wt} \% \mathrm{mHNTs}$ & 83 & 3.29 \\
\hline $10 \mathrm{wt} \% \mathrm{mHNTs}$ & 79 & 3.25 \\
\hline
\end{tabular}

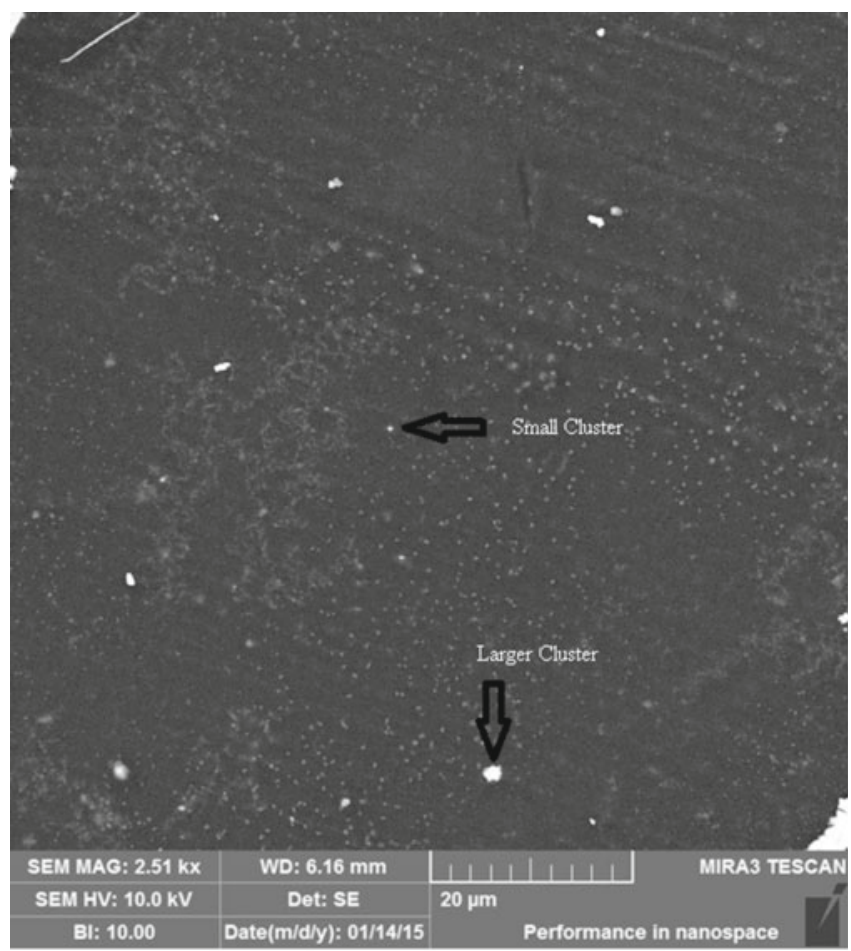

Figure 3. SEM image of HNTs-epoxy composite (10\% loading)

improvement in flexural properties of composites with incorporation HNTs in polymers ${ }^{\mathbf{1 0}}$.

\section{CONCLUSIONS}

Naturally occuring halloysite nanotubes were employed as reinforcement in epoxy polymer matrix. Modified HNTs were prepared by coupling with silane to improve dispersion and interfacial interaction in epoxy matrix. It appears that without modification, HNTs tend to exist in cluster. Prepared nanocomposites showed improved flexural properties, dimensional stability and char yield. This work leads to a better understanding of the role of a relatively cheap and naturally occuring reinforcement material to prepare mechanically improved polymeric composite materials. Due to unique morphology of HNTs, proper allignment of HNTs in polymer matrix can definitely affect the mechanical properties of composites. Further research in surface modificaiton methods can lead to improved anisotropic mechanical properties of HNTs reinforced composite materials.

\section{ACKNOWLEDGEMENTS}

We are thankful to Higher Education Commission Pakistan to provide fund to carry out this research project.

\section{LITERATURE CITED}

1. Kirkman, J.H. (1977). Possible structure of halloysite disks and cylinders observed in some New Zealand rhyolitic tephras. Clay Miner. 3(12), 199-216.

2. Joussein, E., Petit, S., Churchman, J., Theng, B., Righi, D. \& Delvaux, B. (2005). Halloysite clay minerals - a review. Clay Miner. 4(40), 383-426. DOI: 10.1180/0009855054040180.

3. Levis, S.R. \& Deasy, P.B. (2002). Characterisation of halloysite for use as a microtubular drug delivery system. Inter. J. Pharm. 1-2(243), 125-134. DOI: 10.1016/S03785173(02)00274-0.

4. Imai, T., Naitoh, Y., Yamamoto, T. \& Ohyanagi, M. (2006). Translucent Nano Mullite Based Composite Ceramic Fabricated by Spark Plasma Sintering. J. Cer. Soc. JPN 1325(114), 138-140. DOI: 10.2109/jcersj.114.138.

5. Wilson, I.R. (2004). Kaolin and halloysite deposits of China. Clay Miner. 1(39), 1-15. DOI: 10.1180/0009855043910116.

6. Guo, B., Zou, Q., Lei, Y. \& Jia, D. (2009). Structure and Performance of Polyamide 6/Halloysite Nanotubes Nanocomposites. Polym. J. 10(41), 835-842.

7. Handge, U.A., Hedicke-Höchstötter, K. \& Altstädt, V. (2010). Composites of polyamide 6 and silicate nanotubes of the mineral halloysite: Influence of molecular weight on thermal, mechanical and rheological properties. Polymer 12(51), 2690-2699. DOI: http://dx.doi.org/10.1016/j.polymer.2010.04.041.

8. Ning, N., Yin, Q., Luo, F., Zhang, Q., Du, R. \& Fu, Q. (2007). Crystallization behavior and mechanical properties of polypropylene/halloysite composites. Polymer 25(48), 7374-7384. http://dx.doi.org/10.1016/j.polymer.2007.10.005

9. Oburoğlu, N., Ercan, N., Durmus, A. \& Kaşgöz, A. (2011). Effects of Halloysite Nanotube on the Mechanical Properties and Nonisothermal Crystallization Kinetics of Poly(Butylene Terephthalate) (PBT). J. Macromol. Sci., Part B 5(51), 860-879. DOI: $10.1080 / 00222348.2011 .610231$.

10. Szczygielska, A. \& Kijeński, J. (2011). Studies of properties of polypropylene/halloysite composites. Pol. J. Chem. Technol. 3(13), 61. DOI: 10.2478/v10026-011-0039-0. 